\title{
Comparison of cytokine profiles induced by nonlethal and lethal doses of influenza $A$ virus in mice
}

\author{
LUCIA TURIANOVÁ ${ }^{1}$, VERONIKA LACHOVÁ ${ }^{1}$, DARINA SVETLÍKOVA ${ }^{1}$, \\ ANNA KOSTRÁBOVÁ ${ }^{2}$ and TATIANA BETÁKOVÁ ${ }^{1,2}$ \\ ${ }^{1}$ Biomedical Research Center-Slovak Academy of Sciences, Institute of Virology, 84505 Bratislava; \\ ${ }^{2}$ Department of Microbiology and Virology, Faculty of Natural Sciences, \\ Comenius University in Bratislava, 84215 Bratislava, Slovak Republic
}

Received April 5, 2019; Accepted September 9, 2019

DOI: $10.3892 /$ etm.2019.8096

\begin{abstract}
Influenza viruses are among the most common human pathogens and are responsible for causing extensive seasonal morbidity and mortality. To investigate the immunological factors associated with severe influenza infection, the immune responses in mice infected with nonlethal (LD0) doses of $\mathrm{A} / \mathrm{PR} / 8 / 34$ (H1N1) influenza virus were compared with those of mice infected with a lethal dose (LD100) of the virus. The virus titer and activation of retinoic acid-inducible gene (RIG)-I-like receptor signaling pathways were similar in the mice infected with LD0 and LD100 at 2 days post-infection; however, mice infected with LD100 exhibited a greater abundance of cytokines and a more diverse cytokine profile. Infection with LD100 induced the expression of the following factors: Interleukins (ILs), IL-4, IL-7, IL-10, IL-11, IL-12p40, IL-13 and IL-15; inflammatory chemokines, C-C motif chemokine ligand (CCL)2, CCL3/4, CCL12, CCL17, CCL19; and lung injury-associated cytokines, leptin, leukaemia inhibitory factor, macrophage colony stimulating factor, pentraxin (PTX) 2 and PTX3, WNT1-inducible-signaling pathway protein 1, matrix metallopeptidase (MMP)-2, MMP-3, proprotein convertase subtilisin/kexin type 9, and T cell immunoglobulin and mucin domain. Switching in macrophage polarization from M1 to M2 was evidenced by the increase in M2 markers, including arginase-1 (Arg1) and early growth response protein 2 (Egr2), in the lungs of mice infected with LD100. Since IL-12 and interferon- $\gamma$ are the major T helper (Th) 1 cytokines, increased expression of interferon regulatory factor 4, IL-4, IL-10 and IL-13 promoted the differentiation of naïve $\mathrm{CD}^{+} \mathrm{T}$ cells into Th2 cells. In conclusion, the present study identified key cytokines involved in the pathogenicity of influenza infection,
\end{abstract}

Correspondence to: Dr Tatiana Betáková, Biomedical Research Center-Slovak Academy of Sciences, Institute of Virology, Dubravska Cesta 9, 84505 Bratislava, Slovak Republic

E-mail: virubeta@savba.sk

Key words: influenza virus, cytokine, interleukin, chemokine, immune response, lungs, mice and demonstrated that lethal influenza virus infection induces a mixed Th1/Th2 response.

\section{Introduction}

The influenza A virus is a major human pathogen. The severity of infection ranges from mild to severe and may even lead to death. Seasonal viruses can cause annual epidemics that result in 3-5 million cases of severe illness and 290,000-650,000 deaths (1).

In order to effectively diagnose and treat severe influenza virus infections, it is important to determine the infection status and the quality of the host immune response. Therefore, the identification of specific biomarkers that enable accurate diagnosis of the disease and have a prognostic value for predicting disease severity is required (2). Identifying biomarkers of influenza A infection is challenging, as many viral and cellular factors influence virulence, host response and pathogenicity of the virus. Hemagglutinin, neuraminidase, NS and polymerase PB1 and PB2 gene products serve a central role in determination of virulence (3-7). Abundant viral replication in the lungs and dissemination into non-respiratory tract tissues may result in increased pathogenicity and mortality $(7,8)$. Both innate and adaptive immune responses are crucial for the control of influenza infection. The activity of innate and adaptive immune cells is coordinated by cytokines. However, an overactive or unbalanced immune response may result in the overproduction of cytokines that leads to severe inflammation, whereby an excessive number of neutrophils and mononuclear cells are recruited to the site of infection (9). Interleukin (IL)-6 and chemokines, C-C motif chemokine ligand (CCL)2, CCL4, C-X-C motif chemokine ligand (CXCL)8, CXCL9 and CXCL10, are associated with the pathogenicity of both avian (H5N1 and H7N9) and human (pdmH1N1 and H3N2) viruses (10-12). Chemokines, CCL2, CXCL8, CXCL9 and CXCL10, have been associated with mortality (13-17).

In present study, the cytokine expression profile in the lungs of mice infected with a nonlethal dose (LD0) of the A/PR/8/34 virus (H1N1) was analysed and compared with that of mice infected with a lethal dose (LD100) of the same virus. The aim of the present study was to identify the cytokines with altered expression patterns following infection with LD100 when 
compared with LD0. The results provide novel insights into the pathology of influenza A infection and may have applications for the improvement of influenza diagnosis and therapy.

\section{Materials and methods}

Cells and viruses. MDCK (ATCC CCL-34) cells were grown in Dulbecco's modified Eagle's medium (Lonza) containing $10 \%$ fetal calf serum (HyClone Laboratories). Influenza virus $\mathrm{A} / \mathrm{PR} / 8 / 34$ [H1N1] was cultured in 10-days-old fertile hen's eggs.

Female BALB/c mice (age, 4 weeks; body weight approximately $20 \mathrm{~g}$ ) were purchased from the Faculty of Medicine, Masaryk University (Czech Republic). A total of 30 mice in two groups of 15 mice were anesthetized with Zoletil $(5 \mathrm{mg} / \mathrm{kg})$ intraperitoneally and inoculated intranasally with $10^{3}$ plaque-forming units (PFU; LD100) or $10^{1} \mathrm{PFU}$ (LD0) of virus $(40 \mu \mathrm{l})$. To ensure that the mice were fully anesthetized, the monitoring of rear foot reflexes was made before infection, and continual observation of respiratory pattern, mucous membrane colour and responsiveness to manipulations was made throughout the procedure. The mice were monitored daily and humanely sacrificed at the experimental endpoint. The criteria for euthanasia were establish by using the total score for observation of possible animal distress. Weight loss exceeding $25 \%$ of the original body weight, decrease of appetite, weakness, shivering, depression and moribund state of animals were monitored twice per day. For the cytokine assay, all groups of 4 mice were sacrificed by cervical dislocation and the lungs were aseptically collected at 0,2 and 4 days post-infection (p.i.). Experiment lasted for 8 days. Organ homogenates were pooled together, and aliquots were stored at $-80^{\circ} \mathrm{C}$.

Determination of virus titers. Cellular debris was removed from lung tissue samples by centrifuging at $160 \mathrm{xg}$ for $10 \mathrm{~min}$ at $4^{\circ} \mathrm{C}$ and the supernatants were used for virus quantification. The viral titers are expressed as PFU/ml of lung homogenate in MDCK cells using a plaque assay as previously described (18). The results are expressed as the mean of two independent experiments.

Semi-quantitative reverse transcription-polymerase chain reaction (RT-PCR). Total RNA from the lungs was extracted using the SV Total RNA Isolation System (Promega). A total of $400 \mathrm{ng} / \mu 1 \mathrm{RNA}$ was reverse transcribed using random hexanucleotide primers and the MuLV reverse transcriptase (Finnzyme; Thermo Fisher Scientific). Viral transcripts were detected by semi-quantitative RT-PCR as previously described (18). The sequences of all additional primers used for PCR were as follows: Interferon regulatory factor (IRF)3, forward, 5'-GTCCTCAGATCTGGCTATTG-3' and reverse, 5'-GCTTCAGTGGATTTTCTTGG-3'; IRF4, forward, 5'-TCA CTTGTTCGTGGAGCATC-3' and reverse, 5'-TCTGGAGTC AGTGCTGATGG-3'; IRF7, forward, 5'-CCACGGAAAATA GGGAAGAA-3' and reverse, 5'-CATAGGGTTCCTCGT AAACA-3', Arginase 1 (Arg1), forward, 5'-GCAGTGGCT TTAACCTTGGC-3' and reverse, 5'-CTGTGATGCCCCAGA TGGTT-3'; CD38, forward, 5'-TGGCCTTGCTGGAATAGG TG-3' and reverse, 5'-TCATCAAGGTGGGAGCATGG-3'; early growth response protein 2 (Egr2), forward, 5'-ACC TCCTTCCTACCCATCCC-3' and reverse, 5'-ACAGGGAAA
CGGCTTTCGAT-3'; G-protein coupled receptor 18 (Gpr18), forward 5'-TGGCCATCGTACAGCCAAAA-3' and reverse, 5'-GTCGGAGATCTTCAGGCAGG-3'. Formyl peptide receptor 2 (Fpr2) transcripts were detected by using primers as previously decribed (19).

The band intensity of the PCR products was determined using Gene Tools image analysis software (Syngene). $\beta$-actin was used as an internal control to normalize the expression of the mRNA levels between different samples.

Cytokine array. Lung tissue homogenates $(100 \mu \mathrm{l})$ were lysed and the protein concentration was determined using the Pierce BCA Protein assay kit (Thermo Fisher Scientific). Chemokine expression in lung tissue lysates was assessed using the Proteome Profiler Mouse XL Cytokine array kit (R\&D Systems). Signal intensities on autoradiography films were quantified using Gene Tools software (Sygene). The expression levels of cytokines were normalized to the expression level of reference spots. The assay was performed in duplicate to ensure reproducibility of the results.

Statistical analysis. Statistical analysis was performed by comparing control group (noninfected mice) to infected mice or LD0 to LD100, respectively. Data were analyzed using the unpaired Student's t-test for data with two groups and ANOVA followed by Tukey's post hoc test was performed for data with $\geq 2$ groups. P-values $<0.05$ were considered to be significant. Statistical analysis was performed using GraphPad Prism software (https://goodcalculators.com/one-way-anova-calculator/and http://www.graphpad.com/quickcalcs/ttest1.cfm).

\section{Results}

Mortality and virus replication. To determine the impact of viral infection on influenza-specific mortality, BALB/c mice were inoculated with either $10^{1} \mathrm{PFU}$ or $10^{3} \mathrm{PFU}$ of H1N1 virus. All animals rapidly developed severe signs of disease, including ruffled fur, huddling, lethargy and weight loss. Mice infected with LD0 exhibited body weight loss of up to $20 \%$ within 4 days of infection, followed by a gradual recovery by the end of the study (Fig. 1A). By contrast, animals infected with LD100 exhibited a $>25 \%$ loss of body weight, and the aforementioned symptoms were more severe. These mice either died or were euthanized, and the death was recorded as infection-associated mortality (Fig. 1B).

The results demonstrated that macroscopic changes in the lungs were associated with virus pathogenicity. Naked-eye observations of the lungs revealed that the most severe signs of damage were identified in mice infected with LD100 at 4 days p.i. (Fig. 2A). Minimal pathological changes were observed in the lungs from mice in the LD0 group. In the same group, the maximum viral titer was observed at 2 days p.i. and these levels were not altered over the subsequent 2 days (Fig. 2B). At 4 days p.i., the viral titer decreased and was undetectable at 8 days p.i. In LD100-infected mice, the viral titer increased up to 4 days p.i. The maximum viral titer in this group of mice was five times higher than mice in the LD0 group.

Activation of retinoic acid-inducible gene (RIG)-I-like receptor signaling pathway genes. The mRNA levels of 

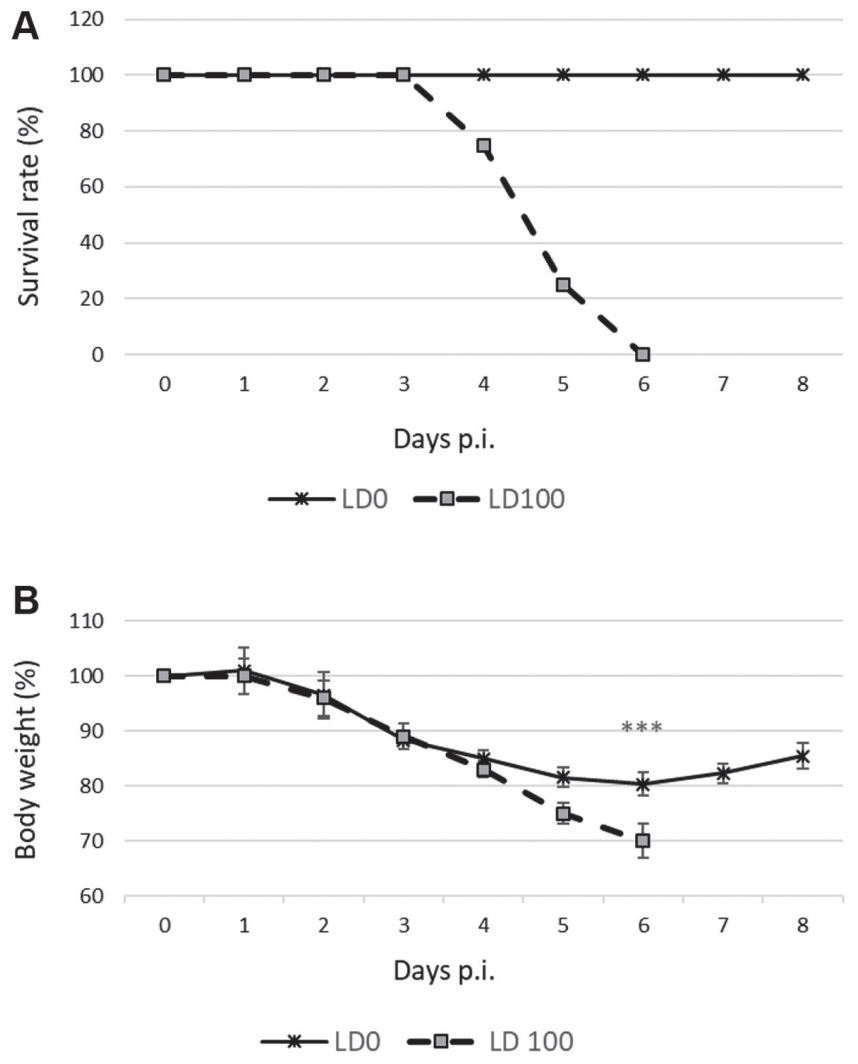

Figure 1. Survival rates of mice following infection with LD0 and LD100 doses of H1N1 virus. BALB/c mice were inoculated intranasally with LD0 and LD100 of H1N1. (A) Percentage body weight loss relative to initial weights was recorded daily until 8 days p.i. (B) Survival rates were calculated as the number of surviving animals/total number of animals $x 100 . n=2 \times 4$ mice at each time-point. ${ }^{* * *} \mathrm{P}<0.001$ vs. LD100. LD0, $10^{1}$ plaque-forming units; LD100, $10^{3}$ plaque-forming units; H1N1, A/PR/8/34 virus; p.i., post-infection.

selected genes in the infected lungs were determined using a semi-quantitative PCR assay at days 0,2 and 4 days p.i. As demonstrated in Fig. 3A and B, LD0 and LD100 doses of the virus induced similar expression alterations of RIG-I and Melanoma differentiation-associated protein 5 (MDA-5) mRNAs. LD100 induced significantly higher levels of RIG-I mRNA at 4 days p.i. No signficant difference in IRF3 and NF-kB mRNA expression levels was observed. IRF7 mRNA levels were significantly increased in mice infected with lethal doses of the virus at 4 days p.i. $(\mathrm{P}<0.05)$. In addition, LD100 induced significant increases in IRF4 mRNA levels at 2 and 4 days p.i. $(\mathrm{P}<0.01$ and $\mathrm{P}<0.05)$. Both viral doses induced similar expression alterations of interferon (IFN) mRNA levels, except for IFN- $\beta$ and IFN- $\varepsilon$. LD0 induced a significant increase in IFN- $\beta$ mRNA levels at 4 days p.i. $(\mathrm{P}<0.05)$. In addition, LD100 induced a significant increase in IFN- $\varepsilon$ mRNA expression at 2 days $(\mathrm{P}<0.05)$ and 4 days $(\mathrm{P}<0.001)$ p.i.

Infection with a lethal dose of virus significantly increased the expression of cytokines associated with pathogenicity. Different cytokine expression profiles were observed following infection with LD0 and LD100. The expression levels of 114 soluble mouse proteins in the lungs of mice infected with either dose of the virus were compared. Both LD0 and LD100 significantly increased the expression levels of lipocalin 2, IL-33, CCL6, CCL11 and CCL22. The expression of these
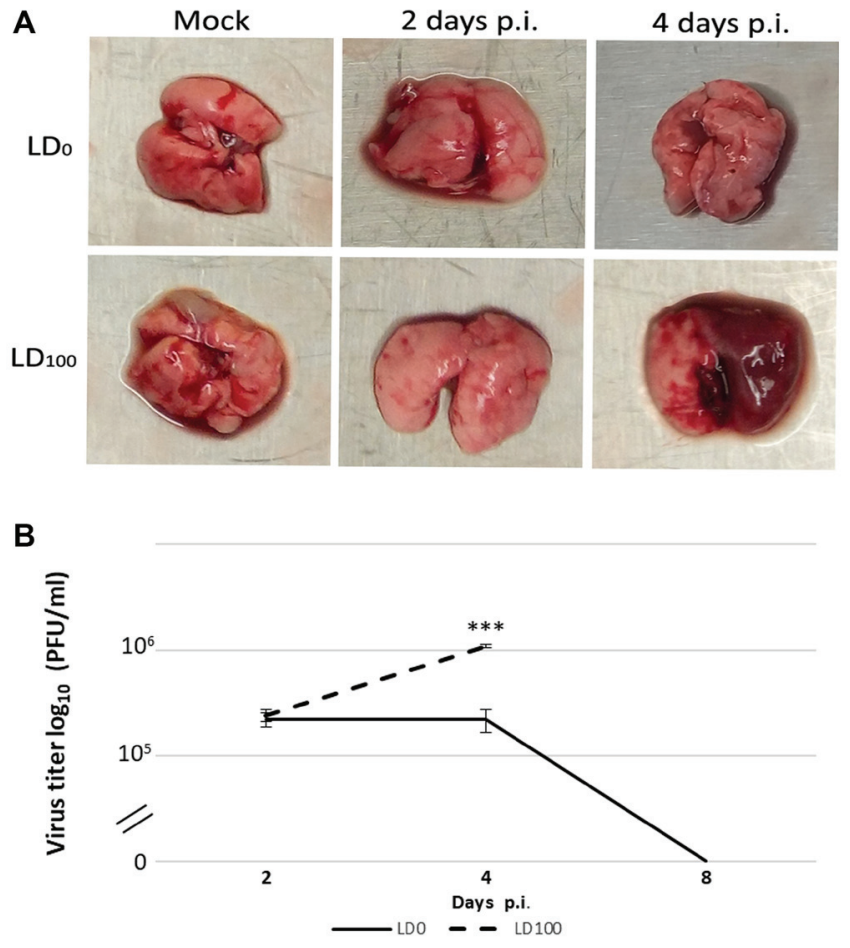

Figure 2. Macroscopic alterations and virus titer in the lungs. Mice were infected with LD0 and LD100 doses of H1N1 influenza virus. (A) Lungs were harvested prior to infection and at 2 and 4 days p.i. (B) Virus titer was determined in lung tissue homogenates. The values represent the mean of two independent experiments. ${ }^{* * *} \mathrm{P}<0.001$ vs. LD0. LD0, $10^{1}$ plaque-forming units; LD100, $10^{3}$ plaque-forming units; H1N1, A/PR/8/34 virus; p.i., post-infection.

cytokines was not positively correlated with the quantity of virus used for infection (data not shown).

LD0 and LD100 viral doses increased the expression of 16 cytokines at 2 and 4 days p.i. (Fig. 4). However, distinct differences in the protein expression patterns of cytokines induced by LD0 and LD100 were observed. Cytokines, IL-1 $\alpha$, CCL5, CXCL5, CXCL9, CXCL10, CXCL11, CXCL13, CXCL16, C-reactive protein (CRP) and matrix metallopeptidase (MMP)-9 were more abundant in the lungs of mice infected with LD100, particularly at 2 days p.i. A significant decrease in the expression of vascular endothelial growth factor (VEGF) and osteopontin (OPN) was observed at 4 days p.i. An additional group of the cytokines, including CXCL5, fetuin A, myeloperoxidase (MPO), plasminogen activator inhibitor type 1 (PAI-1) and low density lipoprotein receptor (LDL-R) were highly induced by LD100 at 2 and 4 days p.i. The expression of these cytokines was significantly higher when compared to LD0 at the same time-points p.i.

Cytokines induced by the lethal dose of virus only. The most apparent differences between the cytokine levels induced by LD0 and LD100 doses of the virus are shown in Fig. 5. LD0 did not influence the expression of these cytokines, whereas LD100 increased their expression at 2 days p.i. These cytokines can be divided into the following three main groups of proteins: i) IFN- $\gamma$; ii) IL-4, IL-7, IL-10, IL-11, IL-12p40, IL-13, IL-15 and chemokines, CCL2, CCL3/4, CCL12, CCL17, CCL19; and iii) other immune response proteins, including leptin, leukaemia inhibitory factor (LIF), macrophage 

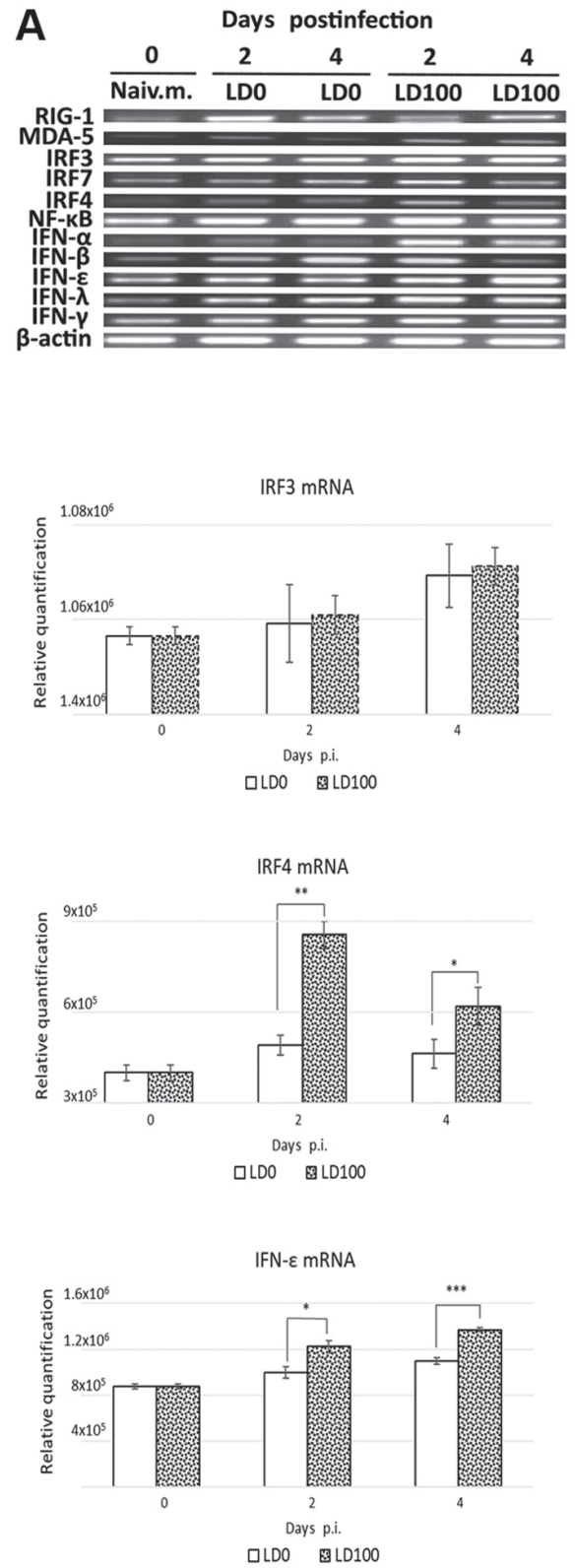

B

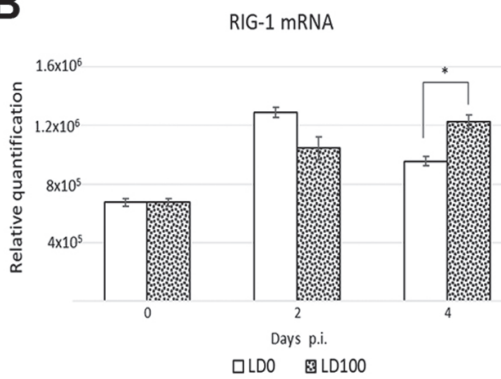

IRF7 mRNA

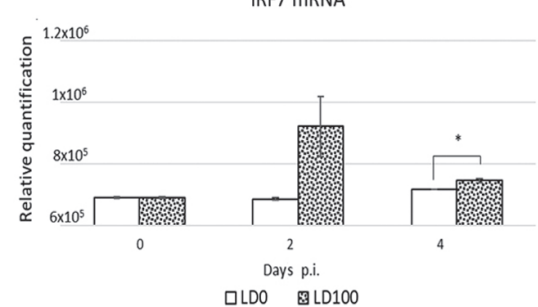

IFN- $\alpha$ mRNA
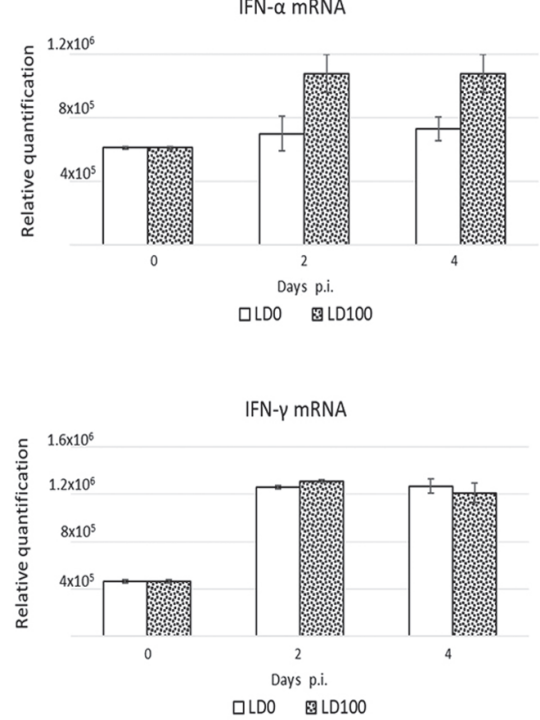
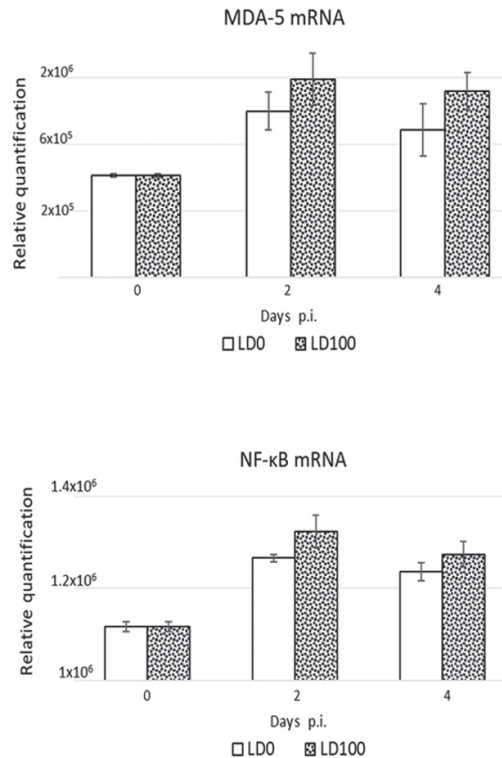

IFN- $\beta$ mRNA

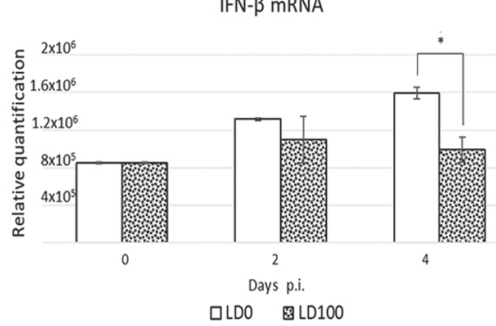

IFN- $\lambda$ mRNA

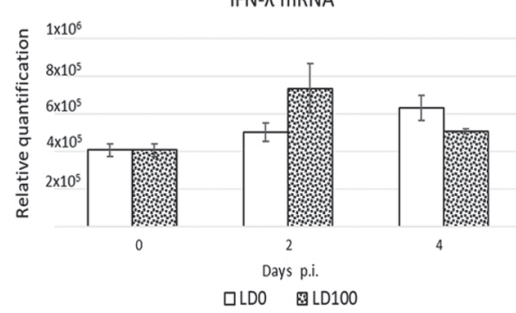

Figure 3. Induction of RIG-I-like receptor signalling pathways. BALB/c mice were infected intranasally with LD0 and LD100 doses of H1N1 virus. The lungs were harvested prior to infection and at 2 and 4 days p.i., and lung homogenates were used for assessment of mRNA levels. Representative (A) reverse transcription PCR blots and (B) relative expression levels of mRNAs were obtained. The expression values represent the mean of two separate experiments and are presented as the mean \pm standard deviation. ${ }^{*} \mathrm{P}<0.05,{ }^{* *} \mathrm{P}<0.001$ and ${ }^{* * *} \mathrm{P}<0.0001$, as indicated. RIG, retinoic acid-inducible gene; LD0, $10^{1}$ plaque-forming units; LD100, $10^{3}$ plaque-forming units; H1N1, A/PR/8/34 virus; IFN, interferon; IRF, interferon regulatory factor; p.i., post-infection; MDA-5, melanoma differentiation-associated gene 5 .

colony stimulating factor (M-CSF), pentraxin (PTX)2 and 3, WNT1-inducible-signaling pathway protein 1 (WISP-1), MMP-2, MMP-3, proprotein convertase subtilisin/kexin type 9 (PCSK9), T cell immunoglobulin and mucin domain (TIM-1).

Infection with a lethal dose of virus significantly increased the expression of M2 macrophage markers. Different transcriptional mRNA profiling in murine macrophages were observed in the lungs infected with with LD0 and LD100. Classically activated macrophages (M1) can be distinquished from alternatively activated macrophages (M2) by their relative expression of CD38, Gpr18, Fpr2 and Arg1, Egr2, respectivelly. As demonstrated in Fig. 6, no signficant difference in CD38,
Gpr18, and Fpr2 mRNA expression levels was observed at 2 and 4 days p.i. Arg1 and Egr 2 mRNA levels were significantly increased in mice infected with lethal doses of the virus at 4 days p.i. These data indicate that infection with LD100 influences macrophage polarization in the lungs.

\section{Discussion}

Cytokines serve an important role in modulating the host immune response, clearing the virus, and healing any injury caused by the virus. Numerous factors influence the pathogenicity of the virus; thus, it is difficult to identify objective markers that can be used as an effective diagnostic tool and predict disease severity. It is hypothesized that virus 

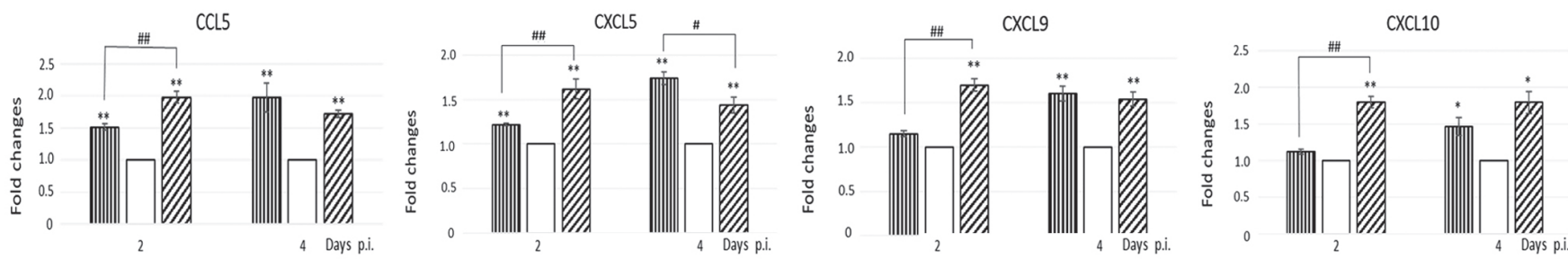

mLDO QMock שLD100

mLD0 aMock $\square L D 100$
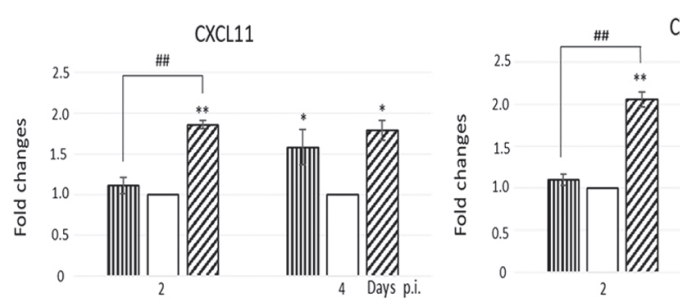

CXCL13
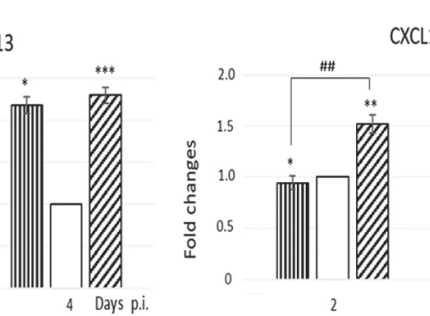

CXCL16

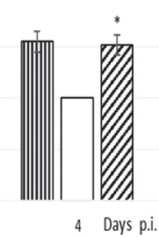

mLDO QMock $\nabla L D 100$

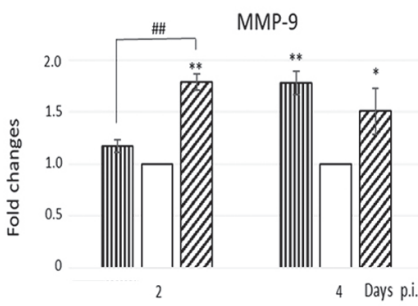

mLDO QMock $₫$ LD100

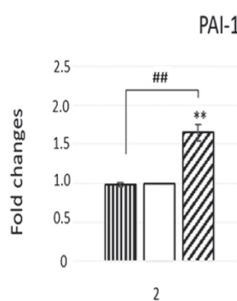

ILDO CMock שLD100
एLDO QMock $\square L D 100$
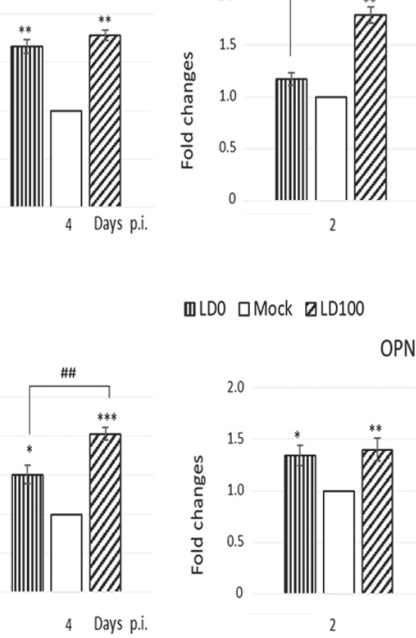

mLDO $\square$ Mock $\square L D 100$

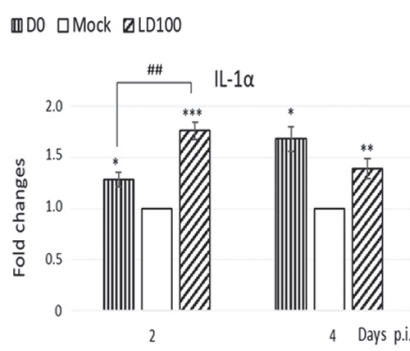

mLD0 aMock వLD100

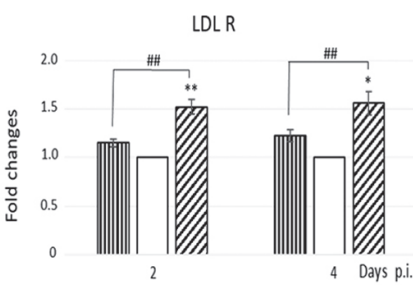

mLD0 QMock LLD100
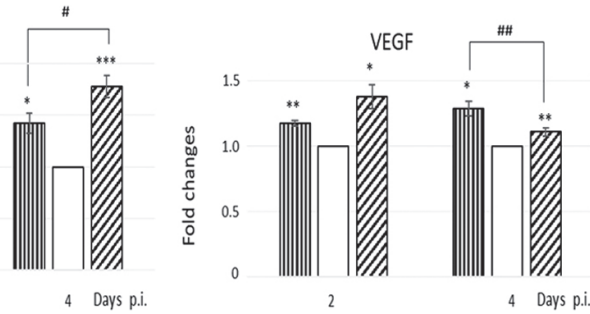

口LDO QMock घLD100

Figure 4. Cytokine expression in the lungs of mice infected with LD0 and LD100 doses of H1N1. Protein expression levels of cytokines in the lungs of infected mice harvested at 2 and 4 days p.i were determined. ${ }^{*} \mathrm{P}<0.05,{ }^{* *} \mathrm{P}<0.001$ and ${ }^{* * *} \mathrm{P}<0.0001$ vs. mock; ${ }^{*} \mathrm{P}<0.05$ and ${ }^{\# \#} \mathrm{P}<0.001$, as indicated. LD0, $10^{1}$ plaque-forming units; LD100, $10^{3}$ plaque-forming units; H1N1, A/PR/8/34 virus; p.i., post-infection.

pathogenicity is directly associated with the replication potential of the virus (7-11). Comparing the expression of cytokines induced by nonlethal and lethal doses of the virus may therefore identify the specific cytokines associated with high pathogenicity, which could be used as suitable biomarkers.

In the present study, the virus titer in mice infected with LD0 and LD100 was similar at 2 days p.i.; however, the cytokine expression profiles induced were different. We repeated this experiment several times and we obtained the same results. As it was expected, $100 \%$ mortality was observed within animals infected with LD100. In LD100-infected mice, the virus titer increased up to 4 days p.i. and none of these mice survived until 8 day p.i. Many factors as virus input, immune response etc. influenced the virus replication in the lungs. The most severe sign of damage was observed in the lungs of mice infected with LD100 at 4 days p.i. Unfortunately, we did not examine histological changes in the lungs after influenza infection. We would like to do it in the future.
No significant difference in the activation of RIG-I-like receptor signaling pathways was observed in the lungs of mice infected with nonlethal and lethal doses of influenza virus. Nevertheless, infection with LD100 induced the expression of a greater abundance and more diverse array of cytokines.

The cytokine profile induced by LD100 was similar to the profiles previously observed in sera from patients with severe influenza infection and influenza virus-associated encephalopathy $(11,12,20)$. IFN- $\gamma$, IL-4, IL-7, IL-10, IL-11, IL-12p40, IL-13 and IL-15 levels were increased only in the lungs of mice infected with a lethal dose of influenza. An interesting observation in our study was a switching in macrophage polarization from M1 to M2, evidenced by the increase in the M2 markers, Arg1 and Egr2, in the lungs of mice infected with LD100. IL-12 and IFN- $\gamma$ are the main Thelper (Th)1 cytokines. Th1 cells emerge in the presence of IL-12 and mediate their function by secreting IFN- $\gamma(21,22)$. Th1 cytokines promote M1 macrophage differentiation, which serves important roles 
A

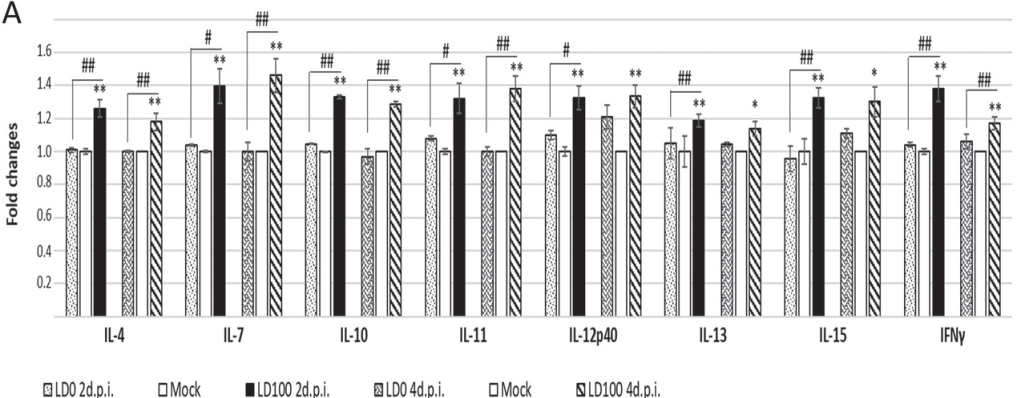

B

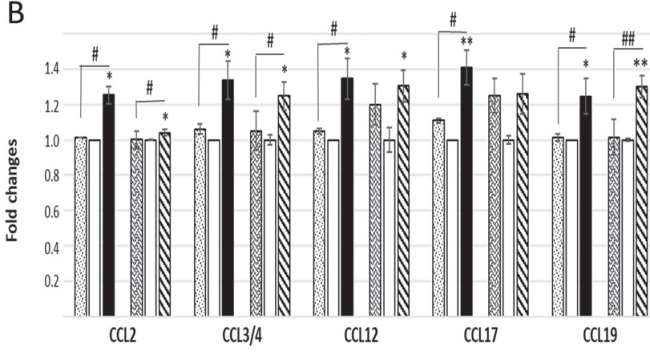

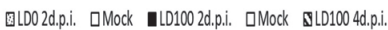

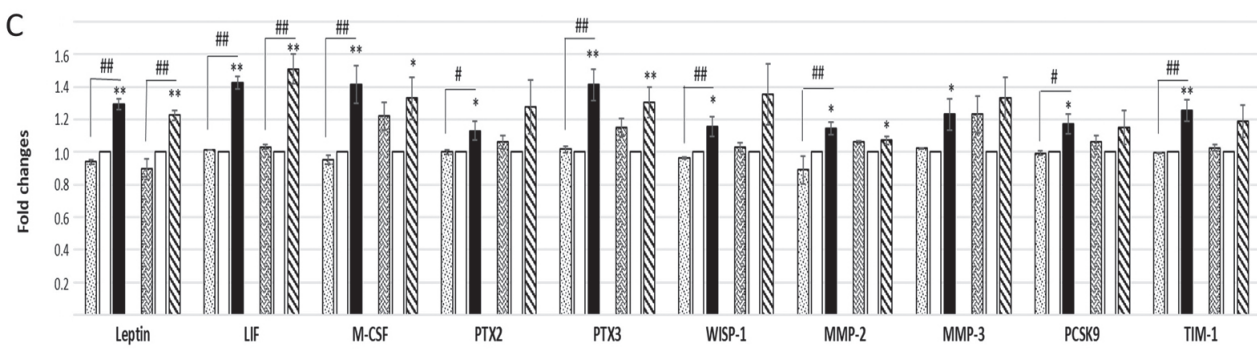

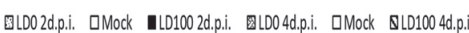

Figure 5. Cytokines exhibiting increased expression in the lungs of the mice infected with a lethal dose of H1N1. BALB/c mice were infected intranasally with LD0 and LD100 doses of H1N1 virus. Protein expression levels of (A) interleukins, (B) chemokines and (C) proteins expressed during tissue damage or injury, were determined in lungs harvested at 2 and 4 days p.i. The values represent the mean of two separate experiments. ${ }^{*} \mathrm{P}<0.05$, and ${ }^{* *} \mathrm{P}<0.001$ vs. mock. ${ }^{*} \mathrm{P}<0.05$ and ${ }^{\# \#} \mathrm{P}<0.001$, as indicated. H1N1, A/PR/8/34 virus; LD0, $10^{1}$ plaque-forming units; LD100, $10^{3}$ plaque-forming units; p.i., post-infection.
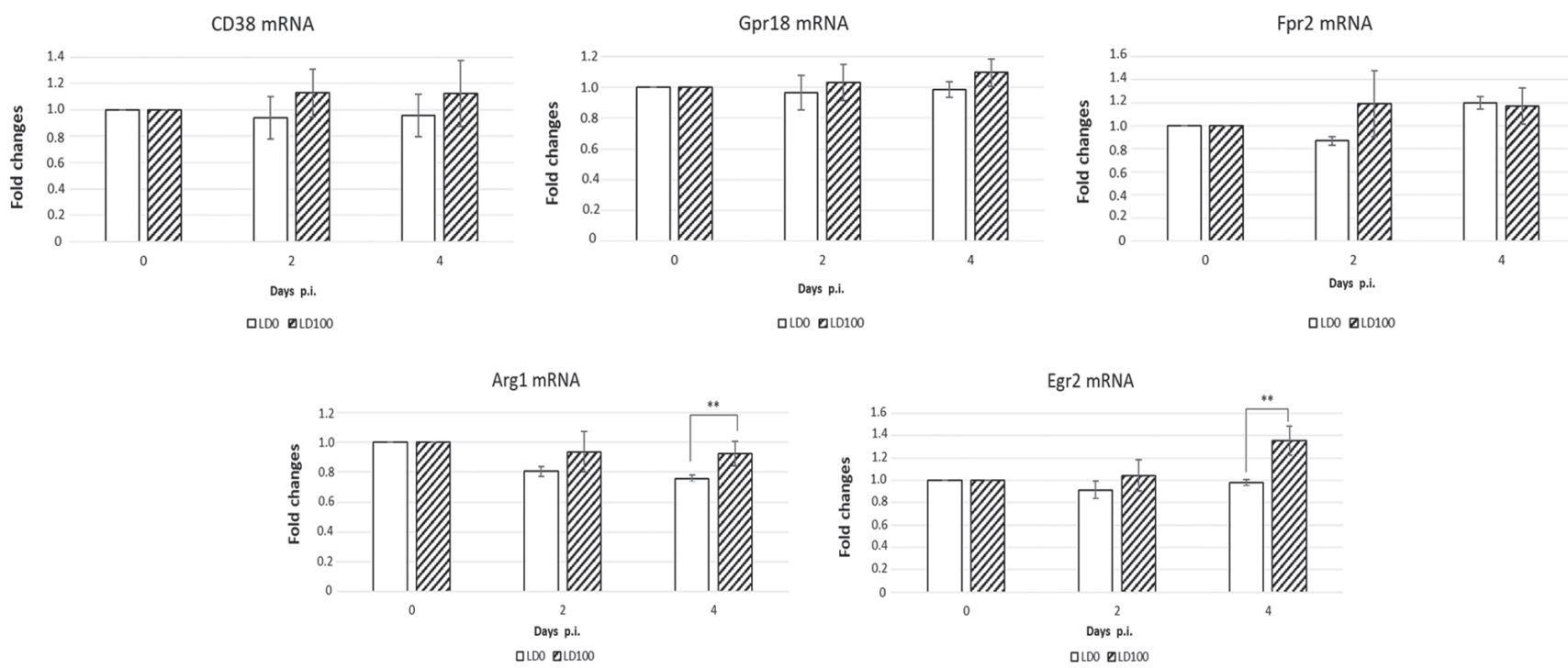

Figure 6. Induction of M21 and M2 markers in the lungs of mice infected with LD0 and LD100 doses of H1N1. The lungs were harvested prior to infection and at 2 and 4 days p.i., and lung homogenates were used for assessment of mRNA levels. Expression values represent the mean of two separate experiments and are expressed as the mean \pm standard deviation. ${ }^{* *} \mathrm{P}<0.001$, as indicated. Gpr18, G-protein coupled receptor 18; Fpr2, formyl peptide receptor 2; Arg1, arginase-1; Egr2, early growth response protein 2; LD0, $10^{1}$ plaque-forming units; LD100, $10^{3}$ plaque-forming units; H1N1, A/PR/8/34 virus; p.i., post-infection.

in the eradication of viral infection. M1 macrophages secrete chemokines, such as CXCL5, CXCL9 and CXCL10 (23). However, excessive pro-inflammatory responses can lead to uncontrolled tissue damage. Anti-inflammatory cytokines, such as IL-4, IL-10 and IL-13 are the main Th2 cytokines. In addition, increased IRF4 and IL-4 levels promote the differentiation of naïve $\mathrm{CD} 4^{+} \mathrm{T}$ cells into $\mathrm{Th} 2$ cells (24-28). Th2 lymphocytes release anti-inflammatory cytokines, IL-4, IL-5, IL-6, IL-10 and IL-13 (29). In the murine system, IL-10 downregulates the Th1 response (30). Subsequent activation of the complement system leads to neutrophil or eosinophil influx by IL-4 and IL-5 $(31,32)$. In addition, Th 2 cytokines, such IL-4 and IL-13, promote differentiation of M2 macrophages. M2 macrophages which produce high levels of IL-10 are generally thought to function as anti-inflammatory macrophages and serve key roles in the suppression of Thl cell responses, wound healing and tissue repair (33-35). Overexpression of chemokines and the Th2 phenotype serve central roles in 
influenza pathogenesis (36). The results of the present study therefore indicate that infection with LD100 invokes a mixed Th1/Th2 response.

In the current study, infection with LD100 induced overexpression of specific $\mathrm{C}$ - $\mathrm{C}$ motif chemokine ligand chemokines, which may contribute to disease pathogenicity and even death of the mice. Overexpression of chemokines CCL2, CCL17 and CCL19 is associated with the activity of M2 macrophages, and serves an important role in protective immune responses (37-39). CCL2 and CCL4 chemokines exert potent effects on the recruitment and degranulation of eosinophils and basophils, which may provide valuable insights into the immunopathogenesis of respiratory viral infections (40). CCL2 and CCL12 mediate acute lung injury induced by lethal influenza infection, as well as by the $\gamma$-herpesvirus or fungal (Asperigillus fumigatus) infections (41-45). However, the precise role of CCL19 in infuenza infection remains unknown. CXCL2 and CCL3 chemokines mediate neutrophil infiltration during the early phase of infection and can serve an important role in the pathogenesis of the disease in the lungs $(46,47)$.

In the current study, increased expression of CRP, LDL-R, MMP-9, MPO, PAI-1, OPN and VEGF correlated with increased inflammatory cytokine expression and lung tissue damage. These proteins are normally expressed during tissue damage or injury and serve important roles in tissue repair and lung immunity. Increased expression of these cytokines has been associated with the pathogenicity of virus infection. MPO-derived oxidants are pathogenic and can promote inflammation and tissue damage (48). Increased MMP-9 expression is associated with organ and tissue damage, and serves an essential role in infection and in the host response to infection (49). The MMP-9 cycle is an important mechanism underlying multiple organ failure during severe influenza infection. A previous study in mouse models demonstrated that H1N1 infection increased the levels of CCL2, MMP-9 and trypsin in serum and/or the lungs and heart (50).

In the present study, infection of mice with a lethal dose of the virus induced the expression of cytokines associated with severe lung injury and pathology. Increased expression of cytokines, including leptin, LIF, M-CSF, PTX2, PTX3, WISP-1, MMP-2, MMP-3, PCSK9 and TIM-1, correlated with extensive lung damage following lethal influenza infection. These proteins modulate infection response, inflammation and tissue repair. A previous study demonstrated that PTX2 reduces the severity of acute lung injury in an animal model and in humans and might be useful for a variety of disease therapy (51). In addition, the IL-10/CREB/WISP-1 signaling pathway has been shown to link innate immune activation to mucosal wound repair (52). MMP-2 and MMP-9 serve a protective role through pathogen clearance (53). However, increased expression of PCSK9 exacerbates multi-organ damage, and MMP3 contributes to the pathogenesis of acute respiratory distress syndrome $(54,55)$. M-CSF promotes the differentiation and survival of macrophages, and preferentially induces anti-inflammatory M2 rather than pro-inflammatory M1 macrophages. Increased levels of C-MSF promote the development of a mixed Th1/Th2 immune response (56). However, it will be necessary to clarify the function of leptin, LIF, M-CSF, PTX2, PTX3, WISP-1, MMP-2, MMP-3, PCSK9 and TIM-1 in influenza pathogenicity. It is possible that these factors are released due to extensive tissue damage caused by viral replication, the increased expression of other cytokines or due to induction of a mixed Th1/Th2 immune response.

The balance between pro- and anti-inflammatory cytokines is essential for maintaining homeostasis in the respiratory system, and an imbalance has been implicated in the pathogenesis of granulomatous diseases and pulmonary fibrosis $(57,58)$. By comparing the cytokine profiles obtained from the lungs of mice infected with nonlethal and lethal influenza doses, the authors of the present study hypothesize that lethal infection may induce a mixed Th1/Th2 response. A previous study demonstrated that Th2-dominated immune responses to influenza virus infection exacerbate lung tissue damage and delay viral clearance (59). In addition, the results of the current study indicate that immune cells, such as macrophages, eosinophils, neutrophils, monocytes, NK cells and basophils may serve a greater role in the pathogenesis of influenza infection than previously thought. However, the mechanisms by which Th1 and Th2 cells influence the inflammatory response during influenza virus infection require further investigation in future studies.

\section{Acknowledgements}

Not applicable.

\section{Funding}

The present study was supported by the Slovak Research and Development Agency (grant nos. APVV-0676-12 and VEGA 2/0014/16).

\section{Availability of data and materials}

The datasets used and/or analyzed during the current study are available from the corresponding author on reasonable request.

\section{Authors' contributions}

TB conceived and designed the experiments, performed experiments, analyzed data and wrote the manuscript. LT and VL performed experiments and analyzed the data. DS performed experiments. AK analyzed data and performed the literature search.

\section{Ethics approval and consent to participate}

All animal experiments were approved by the Institutional Animal Care and Use Committee (IACUC) of the Institute of Virology. The animals were treated according to the European Union standards and the fundamental ethical principles, including animal welfare requirements, were respected. All of the animal experiments were evaluated and approved by the State Veterinary and Food Administration of the Slovak Republic (approval nos. 4370/13-221 and 1204/11-221).

\section{Patient consent for publication}

Not applicable. 


\section{Competing interests}

The authors declare that they have no competing interests.

\section{References}

1. Kalarikkal SM and Jaishankar GB: Influenza Vaccine. StatPearls [Internet]. StatPearls Publishing, Treasure Island, FL, 2019. Available from: https://www.ncbi.nlm.nih.gov/books/ NBK537197/

2. Kollmus H, Pilzner C, Leist SR, Heise M, Geffers R and Schughart K: Of mice and men: The host response to influenza virus infection. Mamm Genome 29: 446-470, 2018.

3. Baskin CR, Bielefeldt-Ohmann H, Tumpey TM, Sabourin PJ, Long JP, García-Sastre A, Tolnay AE, Albrecht R, Pyles JA, Olson PH, et al: Early and sustained innate immune response defines pathology and death in nonhuman primates infected by highly pathogenic influenza virus. Proc Natl Acad Sci USA 106 3455-3460, 2009

4. Lycett SJ, Ward MJ, Lewis FI, Poon AF, Kosakovsky Pond SL and Brown AJ: Detection of mammalian virulence determinants in highly pathogenic avian influenza H5N1 viruses: Multivariate analysis of published data. J Virol 83: 9901-9910, 2009.

5. de Wit E, Kawaoka Y, de Jong MD and Fouchier RA: Pathogenicity of highly pathogenic avian influenza virus in mammals. Vaccine 26 (Suppl 4): D54-D58, 2008.

6. Ping J, Keleta L, Forbes NE, Dankar S, Stecho W, Tyler S, Zhou Y, Babiuk L, Weingartl H, Halpin RA, et al: Genomic and protein structural maps of adaptive evolution of human influenza A virus to increased virulence in the mouse. PLoS One 6: e21740, 2011.

7. Tumpey TM, García-Sastre A, Taubenberger JK, Palese P, Swayne DE, Pantin-Jackwood MJ, Schultz-Cherry S, Solórzano A, Van Rooijen N, Katz JM and Basler CF: Pathogenicity of influenza viruses with genes from the 1918 pandemic virus: Functional roles of alveolar macrophages and neutrophils in limiting virus replication and mortality in mice. J Virol 79: 14933-14944, 2005.

8. de Jong RM, Stockhofe-Zurwieden N, Verheij ES, de Boer-Luijtze EA, Ruiter SJ, de Leeuw OS and Cornelissen LA: Rapid emergence of a virulent PB2 E627K variant during adaptation of highly pathogenic avian influenza $\mathrm{H} 7 \mathrm{~N} 7$ virus to mice. Virol J 10: 276, 2013.

9. Betakova T, Kostrabova A, Lachova V and Turianova L: Cytokines induced during influenza virus infection. Curr Pharm Des 23: 2616-2622, 2017.

10. Chi Y, Zhu Y, Wen T, Cui L, Ge Y, Jiao Y, Wu T, Ge A, Ji H, $\mathrm{Xu} \mathrm{K}$, et al: Cytokine and chemokine levels in patients infected with the novel avian influenza A (H7N9) virus in China. J Infect Dis 208: 1962-1967, 2013.

11. Bradley-Stewart A, Jolly L, Adamson W, Gunson R, Frew-Gillespie C, Templeton K, Aitken C, Carman W, Cameron S and McSharry C: Cytokine responses in patients with mild or severe influenza A(H1N1)pdm09. J Clin Virol 58: 100-107, 2013.

12. Sun G, Ota C, Kitaoka S, Chiba Y, Takayanagi M, Kitamura T, Yamamoto K, Fujie H, Mikami H, Uematsu M, et al: Elevated serum levels of neutrophil elastase in patients with influenza virus-associated encephalopathy. J Neurol Sci 349: 190-195, 2015

13. Ahn MY, Zhang ZG, Tsang W and Chopp M: Endogenous plasminogen activator expression after embolic focal cerebral ischemia in mice. Brain Res 837: 169-176, 1999.

14. Peiris JS, Yu WC, Leung CW, Cheung CY, Ng WF, Nicholls JM Ng TK, Chan KH, Lai ST, Lim WL, et al: Re-emergence of fatal human influenza A subtype H5N1 disease. Lancet 363: 617-619, 2004.

15. Chan MC, Cheung CY, Chui WH, Tsao SW, Nicholls JM, Chan YO, Chan RW, Long HT, Poon LL, Guan Y and Peiris JS: Proinflammatory cytokine responses induced by influenza A (H5N1) viruses in primary human alveolar and bronchial epithelial cells. Respir Res 6: 135, 2005.

16. Walsh KB, Teijaro JR, Rosen H and Oldstone MB: Quelling the storm: Utilization of sphingosine-1-phosphate receptor signaling to ameliorate influenza virus-induced cytokine storm. Immunol Res 51: 15-25, 2011.

17. Vogel AJ, Harris S, Marsteller N, Condon SA and Brown DM: Early cytokine dysregulation and viral replication are associated with mortality during lethal influenza infection. Viral Immunol 27: 214-224, 2014.

18. Svancarova P, Svetlikova D and Betakova T: Synergic and antagonistic effect of small hairpin RNAs targeting the NS gene of the influenza A virus in cells and mice. Virus Res 195: 100-111, 2015.
19. Jablonski KA, Amici SA, Webb LM, Ruiz-Rosado Jde D, Popovich PG, Partida-Sanchez S and Guerau-de-Arellano M: Novel markers to delineate murine M1 and M2 macrophages. PLoS One 10: e0145342, 2015.

20. Davey RT Jr, Lynfield R, Dwyer DE, Losso MH, Cozzi-Lepri A, Wentworth D, Lane HC, Dewar R, Rupert A, Metcalf JA, et al: The association between serum biomarkers and disease outcome in influenza $\mathrm{A}(\mathrm{H} 1 \mathrm{~N} 1) \mathrm{pdm09}$ virus infection: Results of two international observational cohort studies. PLoS One 8: e57121, 2013.

21. Hsieh CS, Macatonia SE, Tripp CS, Wolf SF, O'Garra A and Murphy KM: Development of TH1 $\mathrm{CD}^{+} \mathrm{T}$ cells through IL-12 produced by Listeria-induced macrophages. Science 260 : 547-549, 1993.

22. Macatonia SE, Hosken NA, Litton M, Vieira P, Hsieh CS, Culpepper JA, Wysocka M, Trinchieri G, Murphy KM and O'Garra A: Dendritic cells produce IL-12 and direct the development of Th1 cells from naive CD4 ${ }^{+} \mathrm{T}$ cells. J Immunol 154: 5071-5079, 1995

23. Suzuki K, Meguro K, Nakagomi D and Nakajima H: Roles of alternatively activated M2 macrophages in allergic contact dermatitis. Allergol Int 66: 392-397, 2017.

24. Lohoff M, Mittrücker HW, Prechtl S, Bischof S, Sommer F, Kock S, Ferrick DA, Duncan GS, Gessner A and Mak TW: Dysregulated $\mathrm{T}$ helper cell differentiation in the absence of interferon regulatory factor 4. Proc Natl Acad Sci USA 99: $11808-11812,2002$

25. Rengarajan J, Mowen KA, McBride KD, Smith ED, Singh H and Glimcher LH: Interferon regulatory factor 4 (IRF4) interacts with NFATc2 to modulate interleukin 4 gene expression. J Exp Med 195: 1003-1012, 2002.

26. Staudt V, Bothur E, Klein M, Lingnau K, Reuter S, Grebe N, Gerlitzki B, Hoffmann M, Ulges A, Taube C, et al: Interferon-regulatory factor 4 is essential for the developmental program of T helper 9 cells. Immunity 33: 192-202, 2010.

27. Huber M and Lohoff M: IRF4 at the crossroads of effector T-cell fate decision. Eur J Immunol 44: 1886-1895, 2014.

28. Zhang Y, Zhang Y, Gu W and Sun B: TH1/TH2 cell differentiation and molecular signals. Adv Exp Med Biol 841: 15-44, 2014.

29. Rossi D and Zlotnik A: The biology of chemokines and their receptors. Annu Rev Immunol 18: 217-242, 2000.

30. Del Prete G, De Carli M, Almerigogna F, Giudizi MG, Biagiotti R and Romagnani S: Human IL-10 is produced by both type 1 helper (Th1) and type 2 helper (Th2) T cell clones and inhibits their antigen-specific proliferation and cytokine production. J Immunol 150: 353-360, 1993

31. Kobayashi K, Kaneda K and Kasama T: Immunopathogenesis of delayed-type hypersensitivity. Microsc Res Tech 53: 241-245, 2001.

32. Mukhopadhyay S and Gal AA: Granulomatous lung disease: An approach to the differential diagnosis. Arch Pathol Lab Med 134: 667-690, 2010

33. Mosser DM and Edwards JP: Exploring the full spectrum of macrophage activation. Nat Rev Immunol 8: 958-969, 2008

34. Mantovani A, Biswas SK, Galdiero MR, Sica A and Locati M: Macrophage plasticity and polarization in tissue repair and remodelling. J Pathol 229: 176-185, 2013.

35. Mokarram $\mathrm{N}$ and Bellamkonda RV: A perspective on immunomodulation and tissue repair. Ann Biomed Eng 42: 338-351, 2014.

36. Belperio JA, Dy M, Murray L, Burdick MD, Xue YY, Strieter RM and Keane MP: The role of the Th2 CC chemokine ligand CCL17 in pulmonary fibrosis. J Immunol 173: 4692-4698, 2004.

37. Rangel-Moreno J, Moyron-Quiroz JE, Hartson L, Kusser K and Randall TD: Pulmonary expression of CXC chemokine ligand 13, CC chemokine ligand 19, and CC chemokine ligand 21 is essential for local immunity to influenza. Proc Natl Acad Sci USA 104: 10577-10582, 2007.

38. Huang SS, Banner D, Degousee N, Leon AJ, Xu L, Paquette SG, Kanagasabai T, Fang Y, Rubino S, Rubin B, et al: Differential pathological and immune responses in newly weaned ferrets are associated with a mild clinical outcome of pandemic 2009 H1N1 infection. J Virol 86: 13187-13201, 2012.

39. Hsu AT, Lupancu TJ, Lee MC, Fleetwood AJ, Cook AD, Hamilton JA and Achuthan A: Epigenetic and transcriptional regulation of IL4-induced CCL17 production in human monocytes and murine macrophages. J Biol Chem 293: 11415-11423, 2018.

40. Bonville CA, Rosenberg HF and Domachowske JB: Macrophage inflammatory protein-lalpha and RANTES are present in nasal secretions during ongoing upper respiratory tract infection. Pediatr Allergy Immunol 10: 39-44, 1999. 
41. Blease K, Mehrad B, Lukacs NW, Kunkel SL, Standiford TJ and Hogaboam CM: Antifungal and airway remodeling roles for murine monocyte chemoattractant protein-1/CCL2 during pulmonary exposure to Asperigillus fumigatus conidia. J Immunol 166: 1832-1842, 2001.

42. Vannella KM, Luckhardt TR, Wilke CA, van Dyk LF, Toews GB and Moore BB: Latent herpesvirus infection augments experimental pulmonary fibrosis. Am J Respir Crit Care Med 181: 465-477, 2010.

43. Stoolman JS, Vannella KM, Coomes SM, Wilke CA, Sisson TH, Toews GB and Moore BB: Latent infection by $\gamma$ herpesvirus stimulates profibrotic mediator release from multiple cell types. Am J Physiol Lung Cell Mol Physiol 300: L274-L285, 2011.

44. Lai C, Wang K, Zhao Z, Zhang L, Gu H, Yang P and Wang X: $\mathrm{C}-\mathrm{C}$ motif chemokine ligand 2 (CCL2) mediates acute lung injury induced by lethal influenza H7N9 Virus. Front Microbiol 8: 587, 2017.

45. Wolf S, Johnson S, Perwitasari O, Mahalingam S and Tripp RA Targeting the pro-inflammatory factor CCL2 (MCP-1) with bindarit for influenza A (H7N9) treatment. Clin Transl Immunology 6: e135, 2017.

46. Sakai S, Kawamata H, Mantani N, Kogure T, Shimada Y, Terasawa K, Sakai T, Imanishi $\mathrm{N}$ and Ochiai $\mathrm{H}$ : Therapeutic effect of anti-macrophage inflammatory protein 2 antibody on influenza virus-induced pneumonia in mice. J Virol 74: 2472-2476, 2000.

47. Camp JV, Bagci U, Chu YK, Squier B, Fraig M, Uriarte SM, Guo H, Mollura DJ and Jonsson CB: Lower respiratory tract infection of the ferret by $2009 \mathrm{H} 1 \mathrm{~N} 1$ pandemic influenza A Virus triggers biphasic, systemic, and local recruitment of neutrophils J Virol 89: 8733-8748, 2015.

48. Strzepa A, Pritchard KA and Dittel BN: Myeloperoxidase: A new player in autoimmunity. Cell Immunol 317: 1-8, 2017.

49. Luplertlop N, Missé D, Bray D, Deleuze V, Gonzalez JP, Leardkamolkarn V, Yssel H and Veas F: Dengue-virus-infected dendritic cells trigger vascular leakage through metalloproteinase overproduction. EMBO Rep 7: 1176-1181, 2006.

50. Takahashi E, Indalao IL, Sawabuchi T, Mizuno K, Sakai S, Kimoto $\mathrm{T}, \mathrm{Kim} \mathrm{H}$ and Kido $\mathrm{H}$ : Clarithromycin suppresses induction of monocyte chemoattractant protein-1 and matrix metalloproteinase-9 and improves pathological changes in the lungs and heart of mice infected with influenza A virus. Comp Immunol Microbiol Infect Dis 56: 6-13, 2018.

51. Pilling D and Gomer RH: The development of serum amyloid P as a possible therapeutic. Front Immunol 9: 2328, 2018.
52. Quiros M, Nishio H, Neumann PA, Siuda D, Brazil JC, Azcutia V, Hilgarth R, O'Leary MN, Garcia-Hernandez V, Leoni G, et al: Macrophage-derived IL-10 mediates mucosal repair by epithelial WISP-1 signaling. J Clin Invest 127: 3510-3520, 2017.

53. Hendrix AY and Kheradmand F: The role of matrix metalloproteinases in development, repair, and destruction of the lungs. Prog Mol Biol Transl Sci 148: 1-29, 2017.

54. DwivediDJ,Grin PM, Khan M,Prat A,Zhou J,Fox-Robichaud AE, Seidah NG and Liaw PC: Differential expression of PCSK9 modulates infection, inflammation, and coagulation in a murine model of sepsis. Shock 46: 672-680, 2016.

55. Puntorieri V, McCaig LA, Howlett CJ, Yao LJ, Lewis JF, Yamashita CM and Veldhuizen RA: Lack of matrix metalloproteinase 3 in mouse models of lung injury ameliorates the pulmonary inflammatory response in female but not in male mice. Exp Lung Res 42: 365-379, 2016.

56. Fleetwood AJ, Lawrence T, Hamilton JA and Cook AD: Granulocyte-macrophage colony-stimulating factor (CSF) and macrophage CSF-dependent macrophage phenotypes display differences in cytokine profiles and transcription factor activities: Implications for CSF blockade in inflammation. J Immunol 178: 5245-5252, 2007.

57. da Costa Souza P, Dondo PS, Souza G, Lopes D, Moscardi M, de Miranda Martinho V, de Mattos Lourenço RD, Prieto T, Balancin ML, Assato AK, et al: Comprehensive analysis of immune, extracellular matrices and pathogens profile in lung granulomatosis of unexplained etiology. Hum Pathol 75: 104-115, 2018.

58. Wu C, Luo Z, Pang B, Wang W, Deng M, Jin R, Muhataer X, $\mathrm{Li} \mathrm{Y,} \mathrm{Li} \mathrm{Q} \mathrm{and} \mathrm{Yang} \mathrm{X:} \mathrm{Associations} \mathrm{of} \mathrm{pulmonary} \mathrm{fibrosis}$ with peripheral blood Th1/Th2 cell imbalance and EBF3 gene methylation in uygur pigeon breeder's lung patients. Cell Physiol Biochem 47: 1141-1151, 2018.

59. Graham MB, Braciale VL and Braciale TJ: Influenza virus-specific $\mathrm{CD}^{+} \mathrm{T}$ helper type $2 \mathrm{~T}$ lymphocytes do not promote recovery from experimental virus infection. J Exp Med 180: 1273-1282, 1994.

(i) (9) This work is licensed under a Creative Commons CY NG ND Attribution-NonCommercial-NoDerivatives 4.0 International (CC BY-NC-ND 4.0) License. 